\title{
Ultrasonic Irradiation: Synthesis, Characterization, and Preliminary Antimicrobial Activity of Novel Series of 4,6-Disubstituted-1,3,5-triazine Containing Hydrazone Derivatives
}

\author{
Hessa H. Al-Rasheed, ${ }^{1}$ Monirah Al Alshaikh, ${ }^{1}$ Jamal M. Khaled, ${ }^{2,3}$ \\ Naiyf S. Alharbi, ${ }^{2}$ and Ayman El-Faham ${ }^{1,4}$ \\ ${ }^{1}$ Department of Chemistry, College of Science, King Saud University, P.O. Box 2455, Riyadh 11451, Saudi Arabia \\ ${ }^{2}$ Department of Botany and Microbiology, College of Science, King Saud University, Riyadh, Saudi Arabia \\ ${ }^{3}$ Department of Biotechnology and Food Technology, Thamar University, Dhamar, Yemen \\ ${ }^{4}$ Chemistry Department, Faculty of Science, Alexandria University, P.O. Box 426, Ibrahimia, Alexandria 12321, Egypt
}

Correspondence should be addressed to Jamal M. Khaled; gkhaled@ksu.edu.sa and

Ayman El-Faham; aymanel_faham@hotmail.com

Received 1 September 2016; Revised 19 October 2016; Accepted 31 October 2016

Academic Editor: Andrea Penoni

Copyright (C) 2016 Hessa H. Al-Rasheed et al. This is an open access article distributed under the Creative Commons Attribution License, which permits unrestricted use, distribution, and reproduction in any medium, provided the original work is properly cited.

Novel series of 4,6-disubstituted-1,3,5-triazines containing hydrazone derivatives were synthesized employing ultrasonic irradiation and conventional heating. The ultrasonication gave the target products in higher yields and purity in shorter reaction time compared with the conventional method. IR, NMR $\left({ }^{1} \mathrm{H}\right.$ and $\left.{ }^{13} \mathrm{C}\right)$, elemental analysis, and LC-MS confirmed the structures of the new products. The antimicrobial and antifungal activities were evaluated for all the prepared compounds against some selected Grampositive and Gram-negative bacterial strains. The results showed that only two compounds $7 \mathbf{i}$ (pyridine derivative) and 7k (4chlorobenzaldehyde derivative) displayed biological activity against some Gram-positive and Gram-negative bacteria, while the rest of the tested compounds did not display any antifungal activity.

\section{Introduction}

Ultrasound has been employed more and more frequently in organic synthesis $[1,2]$, because it improved the reaction rate and could adjust the selectivity performance of the reaction [3]. Comparing with traditional methods, ultrasonic irradiation is suitable and simply controlled and it considered as a green powerful synthetic technique in chemical processes. Ultrasonic irradiation has proven to be a particularly important tool for meeting goals of the green chemistry, which is minimization of waste, and decreasing of the energy required for the reaction [4]. Applications of ultrasonic irradiation in organic synthesis are playing important role, especially in cases where traditional methods require drastic conditions or elongated reaction time [5-10].
Richards and Loomis first reported the chemical effects of ultrasound in 1927 [11]. The effect of ultrasound during organic reaction is due to cavitation, which led to separation of molecules of liquids and then the collapse of the bubbles offers strong impulsions that generate shortlived regions with high pressure and temperature. Such localized hot spots act as microreactors in which the sound energy converted into beneficial chemical form [12-14].

Recently Schiff base hydrazone derivatives have attracted great attention in many applications [15-17]. Compounds bearing hydrazone moiety exhibit a broad range of biological activities, including antifungal, antibacterial, antiviral, antimalarial, antiproliferative, anti-inflammatory, and antipyretic properties [18-22]. 


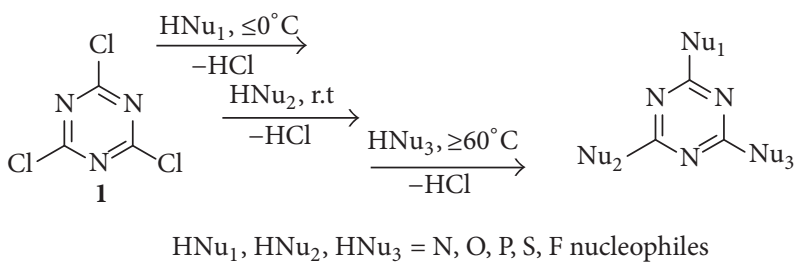

Scheme 1: Nucleophilic substitution reaction of cyanuric chloride.

On the other hand, cyanuric chloride $\mathbf{1}$ is the most important reagent for s-triazine derivatives, because of the selective reactivity of its chlorine atoms toward nucleophiles. Cyanuric chloride $\mathbf{1}$ is commercially available and a very cheap reagent, which makes its applications even more attractive [23-29]. The easy displacement of chlorine atoms in cyanuric chloride by various nucleophiles was controlled by temperature to run in a stepwise manner as shown in Scheme 1.

Several derivatives of s-triazine have exhibited antimicrobial [30], antibacterial, [31] antifungal [32], anti-HIV [33], and anticancer $[34,35]$ properties and a wide array of other biological activities [36, 37]. Recently some of 1,3,5-triazineSchiff bases have reported a significant activity against Mycobacterium tuberculosis H37Rv [38] and moderate to excellent antiproliferative activity and high selectivity against the human lung cancer cell line $\mathrm{H} 460$ [39].

As part of our continuous research on s-triazine derivatives and their biological activities [40,41], we report here the synthesis and the preliminary antimicrobial activity of novel series of 4,6-disubstituted-s-triazine containing hydrazone derivatives.

\section{Experimental Section}

\subsection{Chemistry}

2.1.1. Materials. All solvents were of analytical reagent grade and used without further purification. The ${ }^{1} \mathrm{H}$ NMR and ${ }^{13} \mathrm{C}$ NMR spectra were recorded on a JEOL $400 \mathrm{MHz}$ and AVANCE III $400 \mathrm{MHz}$ (Bruker, Germany) spectrometer at room temperature (see Figures S1-S10, S12, and S14-S17 in Supplementary Material available online at http://dx.doi.org/ $10.1155 / 2016 / 3464758$ ) in $\mathrm{CDCl}_{3}$ and/or $\mathrm{DMSO}^{-\mathrm{d}_{6}}$ using internal standard $\delta=0 \mathrm{ppm}$. Elemental analysis was performed on Perkin-Elmer 2400 elemental analyzer. Melting points were determined on a Mel-Temp apparatus and are uncorrected. Fourier transform infrared spectroscopy (FTIR) spectra were recorded on Nicolet 6700 spectrometer from $\mathrm{KBr}$ discs. The reaction was follow-up and checks of the purity using TLC on silica gel-protected aluminum sheets (Type 60 GF254, Merck) using a mixture of methanolchloroform (1:9) as an eluent. LC-MS (see Figures S11, S13, and S18 in Supplementary Material) was performed on Shimadzu 2020 UFLC-MS using an YMC Triart $C_{18}(5 \mu \mathrm{m}$, $4.6 \times 150 \mathrm{~mm}$ ) column, and data processing was carried out by the LabSolution software. Buffer A: $0.1 \%$ formic acid in $\mathrm{H}_{2} \mathrm{O}$ and buffer B: $0.1 \%$ formic acid in $\mathrm{CH}_{3} \mathrm{CN}$ in $30 \mathrm{~min}$ at $\lambda_{\max }$ $254 \mathrm{~nm}$ were used. High-resolution mass spectrometric data were obtained using a Bruker micrOTOF-Q II instrument operating at room temperature and a sample concentration of approximately $1 \mathrm{ppm}$. Ultrasonic bath was purchased from Selecta (Barcelona, Spain). All compounds were named by using ChemBioDraw Ultra version 14.0, Cambridge Soft Corporation (Cambridge, MA, USA).

(i) General Method for the Synthesis of 2-Hydrazino-4,6-dimorpholinio or Dimethoxy-1,3,5-triazine (3 and 5). Hydrazine hydrate $(10 \mathrm{~mL}, 80 \%)$ was added dropwise to a solution of 2-chloro-4,6-dimorpholino or dimethoxy-1,3,5-triazine 2 or $4(20 \mathrm{mmol})$ in $50 \mathrm{~mL}$ ethanol at room temperature and then the reaction mixture was sonicated for $60 \mathrm{~min}$ at $60^{\circ} \mathrm{C}$. Ethanol and excess hydrazine were removed under vacuum and then excess diethyl ether was added to afford the product as a white solid in yield $>90 \%$. The spectral data of the two products $\mathbf{3}$ and $\mathbf{5}$ were in good agreement with the reported data $[40,42]$ and were used directly without further purification.

(ii) General Method for the Synthesis of 1,3,5-Triazine-hydrazone Derivatives.

Method A: Conventional Method. 2-Hydrazino-4,6-disubstituted-1,3,5-triazine 3 or $5(10 \mathrm{mmol})$ was added to a solution of aldehyde or ketone $\mathbf{6}(10 \mathrm{mmol})$ in ethanol $(30 \mathrm{~mL})$ containing 2-3 drops of acetic acid and then the reaction mixture was stirred under reflux for 3 hours. After completion of the reaction, the solvent was reduced under vacuum and the precipitated product was filtered off, dried at room temperature, and then recrystallized from ethyl acetate to afford the target product (Table 1).

Method B: Ultrasound Assisted Method. A mixture of aldehyde or ketone $6(10 \mathrm{mmol})$ in ethanol $(30 \mathrm{~mL}), 2$-hydrazino-4,6disubstituted-1,3,5-triazine 3 or 5 ( $10 \mathrm{mmol})$, and 2-3 drops of acetic acid in a flask was heated into a sonicator at $40^{\circ} \mathrm{C}$ for 30-60 min. After completion of reaction (TLC, methanolchloroform 1:9) UV lamp was used for spot visualization at $\lambda_{\max } 254$; the solvent was removed under vacuum and the precipitated product was recrystallized from ethyl acetate to afford the target product (Table 1).

2.1.2. (E)-2-(2-Benzylidenehydrazinyl)-4,6-dimethoxy-1,3,5triazine (7a, Supporting Information Figure S1 in Supplementary Material). The product was obtained as a yellow solid in yield $74 \%(\mathrm{~A}), 93 \%(\mathrm{~B}) ; \mathrm{mp} .235-237^{\circ} \mathrm{C}$; IR ( $\mathrm{KBr}$, $\left.\mathrm{cm}^{-1}\right)$ : $3219(\mathrm{NH}), 1561(\mathrm{C}=\mathrm{N}), 1467,1386(\mathrm{C}=\mathrm{C}) ;{ }^{1} \mathrm{H}$ NMR $\left(\right.$ DMSO- $\left._{6}\right): \delta=3.89\left(\mathrm{~s}, 6 \mathrm{H}, 2 \mathrm{OCH}_{3}\right), 7.41-7.43(\mathrm{~m}, 3 \mathrm{H}$, $\mathrm{Ar}), 7.66(\mathrm{~d}, 2 \mathrm{H}, J=6.4 \mathrm{~Hz}, \mathrm{Ar}), 8.17(\mathrm{~s}, 1 \mathrm{H}, \mathrm{CH}), 11.68(\mathrm{~s}$, $1 \mathrm{H}, \mathrm{NH}) ;{ }^{13} \mathrm{C}$ NMR $\left(\right.$ DMSO- $\left._{6}\right): \delta=54.5,126.8,128.8,129.8$, 134.4, 144.9, 166.3 ppm; Anal. Calc. for $\mathrm{C}_{12} \mathrm{H}_{13} \mathrm{~N}_{5} \mathrm{O}_{2}$ (259.27): C, 55.59; H, 5.05; N, 27.01. Found: C, 55.89; H, 5.16; N, 27.23.

2.1.3. (E)-2-(2-(4-Chlorobenzylidene)hydrazinyl)-4,6-dimethoxy-1,3,5-triazine (7b, Supporting Information Figure S2 in Supplementary Material). The product was obtained as a white solid in yield $71 \%(\mathrm{~A}), 92 \%(\mathrm{~B}) ; \mathrm{mp} .215-217^{\circ} \mathrm{C}$; IR $\left(\mathrm{KBr}, \mathrm{cm}^{-1}\right)$ : $3228(\mathrm{NH}), 1614(\mathrm{C}=\mathrm{N}), 1577,1473(\mathrm{C}=\mathrm{C}) ;{ }^{1} \mathrm{H}$ 
TABLE 1: Yield, reaction time, and melting point of $7 \mathbf{a}-\mathbf{o}$ derivativesusing conventional method and ultrasonic irradiation.

\begin{tabular}{|c|c|c|c|c|c|}
\hline \multirow[t]{2}{*}{$\begin{array}{l}\text { Compd. } \\
\text { number }\end{array}$} & \multicolumn{2}{|c|}{$\begin{array}{l}\text { Method A } \\
\text { Conventional } \\
\text { method }\end{array}$} & \multicolumn{2}{|c|}{$\begin{array}{l}\text { Method B } \\
\text { Ultrasonic } \\
\text { irradiation }\end{array}$} & \multirow[t]{2}{*}{$\mathrm{Mp}\left({ }^{\circ} \mathrm{C}\right)$} \\
\hline & Yield (\%) & Time (h) & Yield (\%) & $\begin{array}{l}\text { Time } \\
(\mathrm{min})\end{array}$ & \\
\hline $7 a$ & 74 & $4-5$ & 93 & 30 & $235-237$ \\
\hline $7 b$ & 71 & $4-5$ & 92 & 30 & $215-217$ \\
\hline $7 c$ & 70 & $4-5$ & 93 & 30 & 204-206 \\
\hline $7 d$ & 68 & $4-5$ & 93 & 30 & $188-190$ \\
\hline $7 e$ & 75 & $4-5$ & 95 & 30 & $220-222$ \\
\hline $7 f$ & 75 & $4-5$ & 93 & 60 & $225-227$ \\
\hline $7 g$ & 75 & $4-5$ & 93 & 60 & $227-229$ \\
\hline $7 \mathrm{~h}$ & 71 & $4-5$ & 92 & 60 & $230-232$ \\
\hline $7 \mathbf{i}$ & 71 & $4-5$ & 92 & 30 & $212-214$ \\
\hline $7 \mathbf{j}$ & 75 & $4-5$ & 95 & 30 & $225-227$ \\
\hline $7 k$ & 70 & $4-5$ & 94 & 30 & $233-235$ \\
\hline 71 & 72 & $4-5$ & 94 & 60 & $236-238$ \\
\hline $7 \mathrm{~m}$ & 74 & $4-5$ & 96 & 60 & $213-215$ \\
\hline $7 \mathrm{n}$ & 70 & $4-5$ & 92 & 60 & $228-230$ \\
\hline 70 & 71 & $4-5$ & 90 & 60 & $232-234$ \\
\hline
\end{tabular}

NMR (DMSO-d $\left.\mathrm{d}_{6}\right): \delta=3.89\left(\mathrm{~s}, 6 \mathrm{H}, 2 \mathrm{OCH}_{3}\right), 7.47(\mathrm{~d}, 2 \mathrm{H}, J=$ $8.8 \mathrm{~Hz}, \mathrm{Ar}$ ), 7.69 (d, 2H, J = 8.8 Hz, Ar), 8.15 (s, 1H, CH), 11.73 $(\mathrm{s}, 1 \mathrm{H}, \mathrm{NH}) ;{ }^{13} \mathrm{C}$ NMR (DMSO-d $\left.\mathrm{d}_{6}\right): \delta=54.5,128.4,128.9$, 133.4, 134.1, 143.6, 166.3 ppm; Anal. Calc. for $\mathrm{C}_{12} \mathrm{H}_{12} \mathrm{ClN}_{5} \mathrm{O}_{2}$ (293.07): C, 49.07; H, 4.12; N, 23.84. Found: C, 48.92; H, 4.22; N, 24.03.

2.1.4. (E)-2,4-Dimethoxy-6-(2-(4-methylbenzylidene)hydrazinyl)-1,3,5-triazine (7c, Supporting Information Figure S3 in Supplementary Material). The product was obtained as a light yellow solid in yield 70\% (A), 93\% (B); mp. 204-206 C; IR $\left(\mathrm{KBr}, \mathrm{cm}^{-1}\right): 3219(\mathrm{NH}), 1564(\mathrm{C}=\mathrm{N}), 1465,1367(\mathrm{C}=\mathrm{C}) ;{ }^{1} \mathrm{H}$ NMR (DMSO-d $\left.{ }_{6}\right): \delta=2.33\left(\mathrm{~s}, 3 \mathrm{H}, \mathrm{CH}_{3}\right), 3.90(\mathrm{~s}, 6 \mathrm{H}, 2$ $\left.\mathrm{OCH}_{3}\right), 7.24(\mathrm{~d}, 2 \mathrm{H}, J=8.0 \mathrm{~Hz}, \mathrm{Ar}), 7.58(\mathrm{~d}, 2 \mathrm{H}, J=8.08 \mathrm{~Hz}$, Ar), 8.15 (s, 1H, CH), 11.62 (s, 1H, NH); ${ }^{13}$ C NMR (DMSO- $\mathrm{d}_{6}$ ): $\delta=21.0,54.5,126.8,129.4,131.7,139.6,145.1,166.2$ ppm; Anal. Calc. for: $\mathrm{C}_{13} \mathrm{H}_{15} \mathrm{~N}_{5} \mathrm{O}_{2}$ (273.12): C, 57.13; H, 5.53; N, 25.63. Found: C, 57.33; H, 5.41; N, 25.80.

2.1.5. (E)-2,4-Dimethoxy-6-(2-(4-methoxybenzylidene)hydrazinyl)-1,3,5-triazine (7d, Supporting Information Figure S4 in Supplementary Material). The product was obtained as a light yellow solid in yield 68\% (A), 93\% (B); mp. $188-190^{\circ} \mathrm{C}$; IR $\left(\mathrm{KBr}, \mathrm{cm}^{-1}\right): 3244(\mathrm{NH}), 1570(\mathrm{C}=\mathrm{N}), 1479,1364(\mathrm{C}=\mathrm{C})$; ${ }^{1} \mathrm{H}$ NMR $\left(\mathrm{DMSO}_{-} \mathrm{d}_{6}\right): \delta=3.80\left(\mathrm{~s}, 3 \mathrm{H}, \mathrm{OCH}_{3}\right), 3.91(\mathrm{~s}$, $\left.6 \mathrm{H}, 2 \mathrm{OCH}_{3}\right), 7.04(\mathrm{~d}, 2 \mathrm{H}, J=8.0 \mathrm{~Hz}, \mathrm{Ar}), 7.62(\mathrm{~d}, 2 \mathrm{H}, J=$ $8.08 \mathrm{~Hz}, \mathrm{Ar}), 8.12$ (s, 1H, CH), $11.56(\mathrm{~s}, 1 \mathrm{H}, \mathrm{NH}) ;{ }^{13} \mathrm{C}$ NMR $\left(\right.$ DMSO $\left._{6}\right): \delta=54.5,55.3,114.3,127.0,128.5,130.0,144.9$, 160.6, 166.1 ppm; Anal. Calc for $\mathrm{C}_{13} \mathrm{H}_{15} \mathrm{~N}_{5} \mathrm{O}_{3}$ (289.30): C, 53.97; H, 5.23; N, 24.21. Found: C, 53.73; H, 5.07; N, 24.00.
2.1.6. (E)-2-(2-(4-Bromobenzylidene)hydrazinyl)-4,6-dimethoxy-1,3,5-triazine (7e, Supporting Information Figure S5 in Supplementary Material). The product was obtained as a white solid in yield 75\% (A), 95\% (B); mp. 220-222 C; IR $\left(\mathrm{KBr}, \mathrm{cm}^{-1}\right): 3217(\mathrm{NH}), 1578(\mathrm{C}=\mathrm{N}), 1460,1368(\mathrm{C}=\mathrm{C}) ;{ }^{1} \mathrm{H}$ NMR (DMSO-d $\left.\mathrm{d}_{6}\right): \delta=3.89\left(\mathrm{~s}, 6 \mathrm{H}, 2 \mathrm{OCH}_{3}\right), 7.64(\mathrm{~s}, 4 \mathrm{H}, \mathrm{Ar})$, 8.15 (s, 1H, CH), 11.74 (s, 1H, NH); ${ }^{13} \mathrm{C}$ NMR (DMSO-d 6 ): $\delta=54.5,122.9,128.7,131.87,133.7,143.8,166.3$ ppm; Anal. Calc. for $\mathrm{C}_{12} \mathrm{H}_{12} \mathrm{BrN}_{5} \mathrm{O}_{2}$ (338.17): C, 42.62; H, 3.58; N, 20.71. Found: C, 42.88; H, 3.64; N, 20.98.

2.1.7. (E)-4-((2-(4,6-Dimethoxy-1,3,5-triazin-2-yl)hydrazono)methyl) phenol ( $7 f$, Supporting Information Figure S6 in Supplementary Material). The product was obtained as a white solid in yield 75\% (A), 93\% (B); mp. 225-227 C; IR $\left(\mathrm{KBr}, \mathrm{cm}^{-1}\right): 3229(\mathrm{OH}), 3135(\mathrm{NH}), 1609(\mathrm{C}=\mathrm{N}), 1567,1493$ $(\mathrm{C}=\mathrm{C}) ;{ }^{1} \mathrm{H}$ NMR $\left(\mathrm{DMSO}_{-} \mathrm{d}_{6}\right): \delta=3.88\left(\mathrm{~s}, 3 \mathrm{H}, \mathrm{OCH}_{3}\right), 3.90$ $\left(\mathrm{s}, 3 \mathrm{H}, \mathrm{OCH}_{3}\right), 6.81(\mathrm{~d}, 2 \mathrm{H}, J=8.0 \mathrm{~Hz}, \mathrm{Ar}), 7.52(\mathrm{~d}, 2 \mathrm{H}, J=$ $8.0 \mathrm{~Hz}, \mathrm{Ar}), 8.07$ (s, 1H, CH), 9.93 (s, 1H, OH), 11.47 (s, 1H, $\mathrm{NH}) ;{ }^{13} \mathrm{C}$ NMR $\left(\mathrm{DMSO}_{-} \mathrm{d}_{6}\right): \delta=54.5,115.8,125.5,128.8$, $145.5,159.2,166.1,171.8$ ppm; Anal. Calc. for $\mathrm{C}_{12} \mathrm{H}_{13} \mathrm{~N}_{5} \mathrm{O}_{3}$ (275.27): C, 52.36; H, 4.76; N, 25.44. Found: C, 52.21; H, 4.89; $\mathrm{N}, 25.63$.

2.1.8. (E)-4-((2-(4,6-Dimethoxy-1,3,5-triazin-2-yl)hydrazono)methyl)-N,N-dimethylaniline (7g, Supporting Information Figure S7 in Supplementary Material). The product was obtained as a yellow solid in yield 75\% (A), 93\% (B); mp. 227-229 ${ }^{\circ}$; IR $\left(\mathrm{KBr}, \mathrm{cm}^{-1}\right): 3211(\mathrm{NH}), 1567(\mathrm{C}=\mathrm{N}), 1459$, $1362(\mathrm{C}=\mathrm{C}) ;{ }^{1} \mathrm{H}$ NMR $\left(\mathrm{DMSO}-\mathrm{d}_{6}\right): \delta=2.95\left(\mathrm{~s}, 6 \mathrm{H}, \mathrm{N}\left(\mathrm{CH}_{3}\right)_{2}\right)$, $3.86\left(\mathrm{~s}, 3 \mathrm{H}, \mathrm{OCH}_{3}\right), 3.89\left(\mathrm{~s}, 3 \mathrm{H}, \mathrm{OCH}_{3}\right), 6.71(\mathrm{~d}, 2 \mathrm{H}, J=$ $7.2 \mathrm{~Hz}, \mathrm{Ar}), 7.52$ (d, 2H, J = 8.8 Hz, Ar), 8.03 (s, 1H, CH), $11.38(\mathrm{~s}, 1 \mathrm{H}, \mathrm{NH}) ;{ }^{13} \mathrm{C}$ NMR (DMSO-d $\left.{ }_{6}\right): \delta=40.4,55.0$, $112.5,122.4,128.8,146.6,151.9,166.5$ ppm; Anal. Calc. for $\mathrm{C}_{14} \mathrm{H}_{18} \mathrm{~N}_{6} \mathrm{O}_{2}$ (302.34): C, 55.62; H, 6.00; N, 27.80. Found: C, 55.88; H, 6.20; N, 28.04 .

2.1.9. (E)-4-(1-(2-(4,6-Dimethoxy-1,3,5-triazin-2-yl)hydrazono)ethyl)phenol (7h, Supporting Information Figure S8 in Supplementary Material). The product was obtained as a yellow solid in yield $71 \%$ (A), 92\% (B); mp. 230-232 C; IR ( $\mathrm{KBr}$, $\left.\mathrm{cm}^{-1}\right)$ : $3466(\mathrm{OH}), 3364(\mathrm{NH}), 1576(\mathrm{C}=\mathrm{N}), 1475,1374(\mathrm{C}=\mathrm{C})$; ${ }^{1} \mathrm{H}$ NMR (DMSO-d $\left.{ }_{6}\right): \delta=2.26\left(\mathrm{~s}, 3 \mathrm{H}, \mathrm{CH}_{3}\right), 3.91(\mathrm{~s}, 6 \mathrm{H}$, $\left.2 \mathrm{OCH}_{3}\right), 6.78(\mathrm{~d}, 2 \mathrm{H}, J=8.8 \mathrm{~Hz}, \mathrm{Ar}), 7.68(\mathrm{~d}, 2 \mathrm{H}, J=8.4 \mathrm{~Hz}$, Ar), 9.78 (s, 1H, OH), 10.47 (s, 1H, NH); ${ }^{13} \mathrm{C}$ NMR (DMSO$\left.\mathrm{d}_{6}\right): \delta=13.9,54.4,115.2,127.9,129.2,151.3,158.6,166.9$ ppm; Anal. Calc. for $\mathrm{C}_{13} \mathrm{H}_{15} \mathrm{~N}_{5} \mathrm{O}_{3}$ (289.30): C, 53.97; H, 5.23; N, 24.2. Found: C, 53.77; H, 5.33; N, 24.43.

2.1.10. (E)-2,4-Dimethoxy-6-(2-(pyridin-2-ylmethylene)hydrazinyl)-1,3,5-triazine (7i, Supporting Information Figure S9 in Supplementary Material). The product was obtained as a pale yellow solid in yield $69 \%$ (A), 91\% (B); mp. $212-214^{\circ} \mathrm{C}$; IR $\left(\mathrm{KBr}, \mathrm{cm}^{-1}\right)$ : $3236(\mathrm{NH}), 1572(\mathrm{C}=\mathrm{N}), 1467,1366(\mathrm{C}=\mathrm{C})$; ${ }^{1} \mathrm{H}$ NMR $\left(\mathrm{DMSO}_{-} \mathrm{d}_{6}\right): \delta=3.92\left(\mathrm{~s}, 6 \mathrm{H}, 2 \mathrm{OCH}_{3}\right), 7.39(\mathrm{t}, 1 \mathrm{H}, J$ $=5.6, \mathrm{Ar}), 7.88(\mathrm{t}, 1 \mathrm{H}, J=7.2 \mathrm{~Hz}, \mathrm{Ar}), 7.96(\mathrm{~d}, 1 \mathrm{H}, J=8.0 \mathrm{~Hz}$, Ar), 8.22 (s, 1H, CH), 8.59 (d, $1 \mathrm{H}, J=5.2 \mathrm{~Hz}, \mathrm{Ar}), 11.38$ (s, 1H, 
$\mathrm{NH}) ;{ }^{13} \mathrm{C}$ NMR $\left(\mathrm{DMSO}_{-} \mathrm{d}_{6}\right): \delta=54.1,119.7,123.9,136.9,144.4$, 149.2 , 153.3, 166.5, 171.6 ppm; Anal. Calc. for $\mathrm{C}_{1} \mathrm{H}_{12} \mathrm{~N}_{6} \mathrm{O}_{2}$ (260.26): C, 50.77; H, 4.65; N, 32.29. Found: C, 50.96; H, 4.73; N, 32.40.

2.1.11. (E)-4,4'-(6-(2-Benzylidenehydrazinyl)-1,3,5-triazine2,4-diyl)dimorpholine (7j, Supporting Information Figure S10 in Supplementary Material). The product was obtained as a white solid in yield 75\% (A), 95\% (B); mp. 215-217 C; IR $\left(\mathrm{KBr}, \mathrm{cm}^{-1}\right): 3267(\mathrm{NH}), 1546(\mathrm{C}=\mathrm{N}), 1479,1450(\mathrm{C}=\mathrm{C}) ;{ }^{1} \mathrm{H}$ $\mathrm{NMR}\left(\mathrm{CDCl}_{3}\right): \delta=3.70-3.74\left(\mathrm{~m}, 8 \mathrm{H}, 4 \mathrm{OCH}_{2}-\right), 3.75-3.84$ (m, 8H, $\left.4 \mathrm{NCH}_{2}-\right)$, 7.35-7.41 (m, 3H, Ar), 7.70-7.73 (m, 2H, $\mathrm{Ar}), 7.82(\mathrm{~s}, 1 \mathrm{H}, \mathrm{CH}), 8.31(\mathrm{~s}, 1 \mathrm{H}, \mathrm{NH}) ;{ }^{13} \mathrm{C} \mathrm{NMR}\left(\mathrm{CDCl}_{3}\right): \delta=$ 43.6, 43.7, 66.8, 66.9, 127.1, 128.6, 129.6, 134.2, 142.5, 165.4 ppm; Anal. Calc. for $\mathrm{C}_{18} \mathrm{H}_{23} \mathrm{~N}_{7} \mathrm{O}_{2}$ (369.43): C, 58.52; H, 6.28; N, 26.54. Found: C, 58.74; H, 6.43; N, 26.79. ( $\mathrm{m} / \mathrm{z})$ Calcd: 369.43; LC-MS (M+H) Found: 370 at $R_{t} 14.11$ min (supporting information Figure S11 in Supplementary Material).

2.1.12. (E)-4, $4^{\prime}$-(6-(2-(4-Chlorobenzylidene)hydrazinyl)-1,3,5triazine-2,4-diyl)dimorpholine (7k, Supporting Information Figure S12 in Supplementary Material). The product was obtained as a white solid in yield 70\% (A), 94\% (B); mp. 233-235 ${ }^{\circ}$; IR (KBr, cm $\left.{ }^{-1}\right): 3270(\mathrm{NH}), 1516(\mathrm{C}=\mathrm{N}), 1480$, $1442(\mathrm{C}=\mathrm{C}) ;{ }^{1} \mathrm{H} \mathrm{NMR}\left(\mathrm{CDCl}_{3}\right): \delta=3.71-3.74(\mathrm{~m}, 8 \mathrm{H}, 4$ $\left.\mathrm{OCH}_{2}-\right)$, 3.75-3.84 (m, 8H, $\left.4 \mathrm{NCH}_{2}-\right), 7.36(\mathrm{~d}, 2 \mathrm{H}, J=$ $4.0 \mathrm{~Hz}, \mathrm{Ar}), 7.72(\mathrm{~d}, 2 \mathrm{H}, J=4.0 \mathrm{~Hz}, \mathrm{Ar}), 7.82(\mathrm{~s}, 1 \mathrm{H}, \mathrm{CH})$, 8.31 (brs, $1 \mathrm{H}, \mathrm{NH}) ;{ }^{13} \mathrm{C} \mathrm{NMR}\left(\mathrm{CDCl}_{3}\right): \delta=43.6,43.7,66.8$, $66.9,128.2,128.9,132.8,135.4,141.1,165.4$ ppm; Anal. Calc. for $\mathrm{C}_{18} \mathrm{H}_{22} \mathrm{ClN}_{7} \mathrm{O}_{2}$ (403.87): C, 53.53; H, 5.49; N, 24.28. Found: C, 53.78; H, 5.60; N, 24.51. ( $m / z)$ Calcd: 403.87; LC-MS $(\mathrm{M}+\mathrm{H})$ Found: 404 at $R_{t} 15.8$ min (supporting information Figure S13 in Supplementary Material)

2.1.13. (E)-4, $4^{\prime}$-(6-(2-(4-Bromobenzylidene)hydrazinyl)-1,3,5triazine-2,4-diyl) dimorpholine (7l, Supporting Information Figure S14 in Supplementary Material). The product was obtained as a white solid in yield 72\% (A), 94\% (B); mp 236$238^{\circ} \mathrm{C}$; IR $\left(\mathrm{KBr}, \mathrm{cm}^{-1}\right): 3273(\mathrm{NH}), 1515(\mathrm{C}=\mathrm{N}), 1479,1441$ $(\mathrm{C}=\mathrm{C}) ;{ }^{1} \mathrm{H}$ NMR $\left(\mathrm{DMSO}-\mathrm{d}_{6}\right): \delta=3.62\left(\mathrm{~m}, 8 \mathrm{H}, 4 \mathrm{OCH}_{2}-\right)$, $3.70\left(\mathrm{~m}, 8 \mathrm{H}, 4 \mathrm{NCH}_{2}\right), 7.59$ (s, 4H, Ar), 8.03 (s, 1H, CH), $10.90(\mathrm{~s}, 1 \mathrm{H}, \mathrm{NH}) ;{ }^{13} \mathrm{C} \mathrm{NMR}\left(\mathrm{CDCl}_{3}\right): \delta=43.8,66.6,122.6$, $128.8,132.3,134.9,141.0,164.7,165.3$ ppm; Anal. Calc. for $\mathrm{C}_{18} \mathrm{H}_{22} \mathrm{BrN}_{7} \mathrm{O}_{2}$ (448.33): C, 48.22; H, 4.95; N, 21.87. Found: C, 48.44; H, 5.08; N, 22.03.

2.1.14. (E)-4-((2-(4,6-Dimorpholino-1,3,5-triazin-2-yl)hydrazono)methyl)phenol (7m, Supporting Information Figure S15 in Supplementary Material). The product was obtained as a white solid in yield 74\% (A), 96\% (B); mp 213-215 C; IR ( $\mathrm{KBr}$, $\left.\mathrm{cm}^{-1}\right)$ : $3448(\mathrm{OH}), 3250(\mathrm{NH}), 1543(\mathrm{C}=\mathrm{N}), 1493,1443(\mathrm{C}=\mathrm{C})$; ${ }^{1} \mathrm{H} \mathrm{NMR}\left(\mathrm{CDCl}_{3}\right): \delta=3.62-3.74\left(\mathrm{~m}, 16 \mathrm{H}, 4 \mathrm{~N}-\mathrm{CH}_{2}-\mathrm{CH}_{2}-\mathrm{O}\right)$, $6.74(\mathrm{~d}, 2 \mathrm{H}, J=8.56 \mathrm{~Hz}, \mathrm{Ar}), 7.51(\mathrm{~d}, 2 \mathrm{H}, J=8.60 \mathrm{~Hz}, \mathrm{Ar}), 7.68$ $(\mathrm{s}, 1 \mathrm{H}, \mathrm{CH}), 8.02$ (brs, $1 \mathrm{H}, \mathrm{NH}) ;{ }^{13} \mathrm{C} \mathrm{NMR}\left(\mathrm{CDCl}_{3}\right): \delta=43.6$, 43.7, 66.8, 66.9, 115.6, 126.8, 128.9, 157.4, 165.4 ppm; Anal. Calc. for $\mathrm{C}_{18} \mathrm{H}_{23} \mathrm{~N}_{7} \mathrm{O}_{3}$ (385.43): C, 56.09; H, 6.02; N, 25.44. Found: C, 56.23; H, 6.21; N, 25.69.
2.1.15. (E)-4-((2-(4,6-Dimorpholino-1,3,5-triazin-2-yl)hydrazono)methyl)-N,N-dimethylaniline (7n, Supporting Information Figure 16 in Supplementary Material). The product was obtained as a yellow solid in yield 70\% (A), 92\% (B); mp 228-230 ${ }^{\circ}$; IR $\left(\mathrm{KBr}, \mathrm{cm}^{-1}\right): 3277(\mathrm{NH}), 1529(\mathrm{C}=\mathrm{N}), 1490$, $1441(\mathrm{C}=\mathrm{C}) ;{ }^{1} \mathrm{H}$ NMR $\left(\mathrm{DMSO}_{-} \mathrm{d}_{6}\right): \delta=3.62-3.69(\mathrm{~m}, 16 \mathrm{H}, 4$ $\left.\mathrm{N}-\mathrm{CH}_{2}-\mathrm{CH}_{2}-\mathrm{O}\right), 6.72(\mathrm{~d}, 2 \mathrm{H}, J=8.04 \mathrm{~Hz}, \mathrm{Ar}), 7.44$ (d, 2H, $J$ $=8.8 \mathrm{~Hz}, \mathrm{Ar}), 7.95$ (s, 1H, CH), 10.52 (s, 1H, NH); ${ }^{13} \mathrm{C} \mathrm{NMR}$ $\left(\right.$ DMSO- $\left._{6}\right): \delta 43.8,66.6,112.4,123.2,128.2,143.3,151.4,164.5$, 165.3 ppm; Anal. Calc. for $\mathrm{C}_{20} \mathrm{H}_{28} \mathrm{~N}_{8} \mathrm{O}_{2}$ (412.50): C, 58.24; $\mathrm{H}$, $6.84 ; \mathrm{N}, 27.17$. Found: C, 58.51; H, 6.99; N, 27.36.

2.1.16. (E)-4-(1-(2-(4,6-Dimorpholino-1,3,5-triazin-2-yl)hydrazono)ethyl)phenol (7o, Supporting Information Figure S17 in Supplementary Material). The product was obtained as a white solid in yield $71 \%$ (A), 90\% (B); mp.232-234 C; IR $\left(\mathrm{KBr}, \mathrm{cm}^{-1}\right): 3452(\mathrm{OH}), 3350(\mathrm{NH}), 1563(\mathrm{C}=\mathrm{N}), 1524,1487$ $(\mathrm{C}=\mathrm{C}) ;{ }^{1} \mathrm{H}$ NMR $\left(\mathrm{CDCl}_{3}\right): \delta=2.24\left(\mathrm{~s}, 3 \mathrm{H}, \mathrm{CH}_{3}\right), 3.70-3.83$ (m, $\left.16 \mathrm{H}, 4 \mathrm{~N}-\mathrm{CH}_{2}-\mathrm{CH}_{2}-\mathrm{O}\right), 6.80$ (d, $\left.2 \mathrm{H}, \mathrm{J}=8.8 \mathrm{~Hz}, \mathrm{Ar}\right), 7.69$ (d, $2 \mathrm{H}, J=8.46 \mathrm{~Hz}, \mathrm{Ar}), 7.91$ (s, 1H, CH), 8.01 (brs, 1H, NH); ${ }^{13} \mathrm{C} \mathrm{NMR}\left(\mathrm{CDCl}_{3}\right): \delta=12.7,43.7,66.9,115.3,127.8,128.2$, $130.9,146.9,156.8,164.7,165.2,165.4$ ppm; Anal. Calc. for $\mathrm{C}_{19} \mathrm{H}_{25} \mathrm{~N}_{7} \mathrm{O}_{3}$ (399.46): C, 57.13; H, 6.31; N, 24.55. Found: C, 57.35; H, 6.44; N, 24.80. ( $\mathrm{m} / z)$ Calcd: 399.46; LC-MS $(\mathrm{M}+\mathrm{H})$ Found: 400 at $R_{t} 13.4$ min (supporting information Figure S18 in Supplementary Material)

\subsection{Biology}

2.2.1. Antimicrobial Activity. The antimicrobial activities of all compounds $7 \mathbf{a}-\mathbf{o}$ were evaluated against some selected pathogenic Gram-positive, Gram-negative, and filamentous fungus strains by using the disc diffusion method [43]. Staphylococcus aureus (ATCC 29213); Neisseria meningitides (ATCC 1302); Streptococcus mutans (ATCC 35668); Escherichia coli (ATCC 25922); Pseudomonas aeruginosa (ATCC 27584); Salmonella typhimurium (ATCC 14028); Brevibacillus laterosporus Wild strain; Candida parapsilosis (ATCC 22019); Cryptococcus neoformans Wild strain; Candida albicans (ATCC 60193), Penicillium chrysogenum (AUMC 9476), Aspergillus niger (AUMC 8777), and Fusarium sp were used in the evaluation test.

Mueller-Hinton agar (Scharlau Microbiology, Spain) was used and prepared according to manufacturer's guidelines, where $1 \mathrm{~L}$ of medium was prepared containing $17.5,1.5$, and $17 \mathrm{gm}$ of peptone, meat infusion solid, starch, and agar, respectively. For preparation of $1 \mathrm{~L}$ of medium, $21 \mathrm{gm}$ of powder was dissolved in $1 \mathrm{~L}$ of distilled water and sterilized at $121^{\circ} \mathrm{C}$ for $15 \mathrm{~min}$ by an autoclave (HL-321, Taiwan). After sterilization and cooling to $50^{\circ} \mathrm{C}$, the medium dispensed in Petri dishes and left to cool down to $25^{\circ} \mathrm{C}$. Then, they were inoculated with the bacterial strains by streaking. In the antifungal test, a potato dextrose agar (PDA) (Scharlau, Spain) was used and prepared according to the manufacturer's directions, where $39 \mathrm{gm}$ PDA dissolved in $1 \mathrm{~L}$ of distilled water and then followed the previously described method.

The microbial inoculation suspensions were prepared in sterile sodium chloride solution $(0.89 \%)$ from activated 


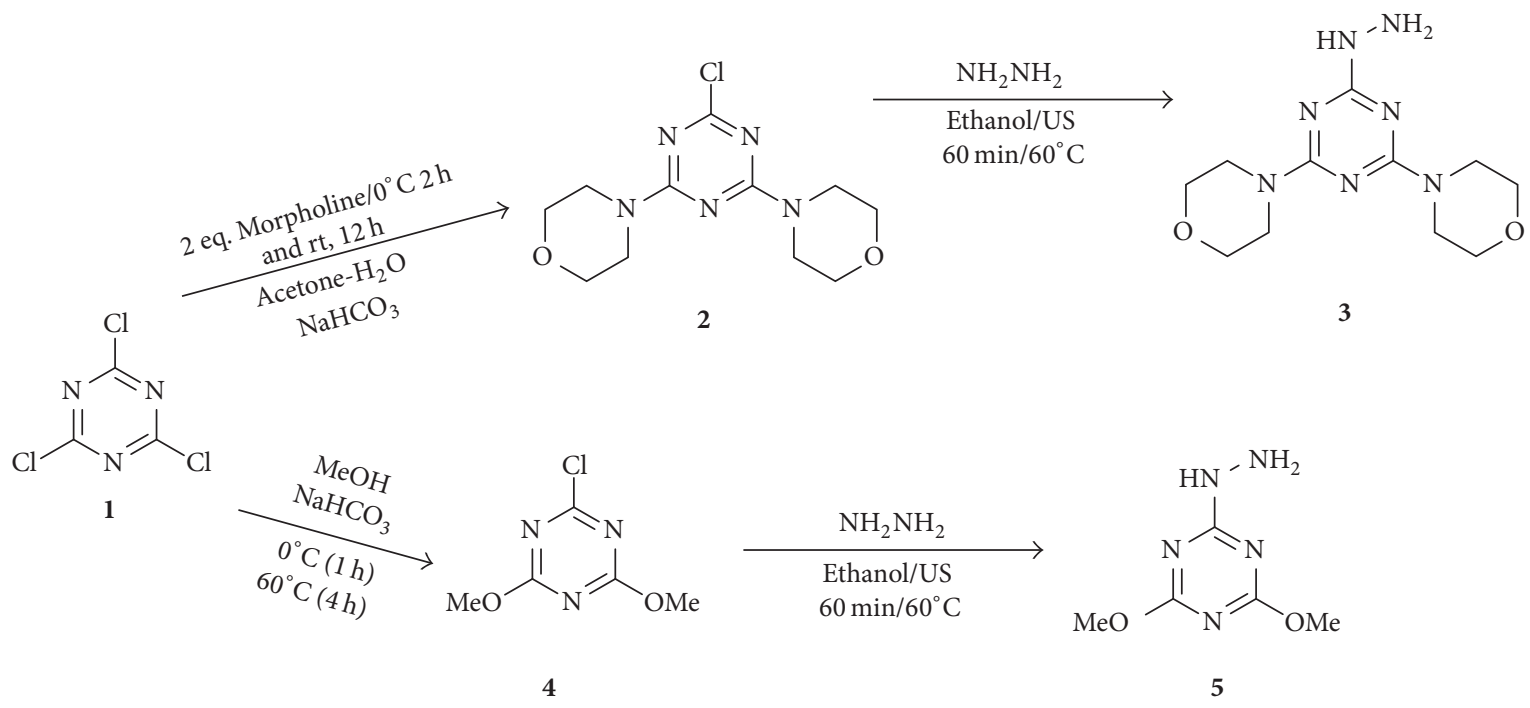

SCHeme 2: Synthesis of 2-hydrazino-4,6-disubstituted-1,3,5-triazine derivatives and US: ultrasonic irradiation.

microbial cultures. The optical densities of microbial suspensions were adjusted to 0.64 at $600 \mathrm{~nm}$. A sterile swab was moistened with the microbial suspensions and inoculating the dried surface of the medium in a Petri dish. After inoculation, all Petri dishes were kept at $25^{\circ} \mathrm{C}$ for 5-10 minutes before dispensing the standard antibiotic agents and the prepared compounds discs on the surface of the media.

In the present work, tobramycin $(10 \mu \mathrm{g} / \mathrm{desk})$, chloramphenicol (30 $\mathrm{g} / \mathrm{disk})$, fusidic acid (10 $\mu \mathrm{g} / \mathrm{disk})$, augmentin $(10 \mu \mathrm{g} / \mathrm{disk})$, cycloheximide $(30 \mu \mathrm{g} / \mathrm{disk})$, canesten $(10 \mu \mathrm{g} /$ disk $)$, and caspofungin $(10 \mu \mathrm{g} / \mathrm{disk})$ were used as standard antibacterial and antifungal agents. To prepare disks ( $4 \mathrm{mg} /$ disk) from the tested compounds, $200 \mathrm{mg}$ from each compound was dissolved in dimethylsulfoxide (DMSO) and then $20 \mu \mathrm{L}$ of the solution was added on the sterile filter disk $(6 \mathrm{~mm})$ and then allowed to dry at room temperature inside the safety biological cabinet. After $18-24 \mathrm{~h}$ of incubation at $37^{\circ} \mathrm{C}$ for bacteria and $48-72 \mathrm{~h}$ at $25^{\circ} \mathrm{C}$ for fungi, the minimum inhibitory concentration ( $\mathrm{MIC} \mathrm{mg} / \mathrm{mL}$ ) of the prepared compounds was measured [44]. The microdilution assay was applied in 96-well plate (Corning Incorporated, USA) using twofold serial dilution. The original concentration was $4 \mathrm{mg} / \mathrm{mL}$ and the total volume was $200 \mu \mathrm{L}(1: 1$, chemical suspension: bacterial suspension); 4-iodonitrotetrazolium violet (Sigma, USA) reagent was added after the incubation to measure the bacterial growth through the emergence of violet color.

\section{Results and Discussion}

3.1. Chemistry. Compounds 2 and $\mathbf{4}$ were obtained using onestep reaction, where cyanuric chloride $\mathbf{1}$ was reacted with morpholine ( 2 equiv.) in acetone-water media or methanol (as a solvent) in the presence of $\mathrm{NaHCO}_{3}$ as hydrogen chloride removal at $0^{\circ} \mathrm{C}$ for $2 \mathrm{~h}$. In the case of the synthesis of dimorpholino derivative $\mathbf{2}$, the reaction temperature raised gradually to room temperature and kept under stirring for $12 \mathrm{~h}$ at the same temperature, while the reported method [40, 42] was used for the preparation of the dimethoxy derivative 4 (Scheme 2). The products 2 and 4 were obtained in good yields and their spectral data agreed with the reported data $[40,42]$.

The hydrazine derivatives 3 and 5 were obtained by treatment of $\mathbf{2}$ or $\mathbf{4}$ with hydrazine hydrate (80\%) in ethanol for $60 \mathrm{~min}$ at $60^{\circ} \mathrm{C}$ employing ultrasonic irradiation (Scheme 2) to afford the products in excellent yields (>90\%) and purity.

The products $7 \mathbf{a}-\mathbf{o}$ were obtained by condensation of the hydrazine derivative 3 or 5 with substituted benzaldehyde or acetophenone 6 in ethanol containing 2-3 drops of glacial acetic acid using ultrasonic irradiation for $30-60 \mathrm{~min}$ at $40^{\circ} \mathrm{C}$ (Scheme 3) to afford the target products in excellent yields and purity as observed from LC-MS (see Supplementary Figures S11, S13, and S18). Ultrasonic irradiation gave the target products in high yields in shorter reaction time compared with the conventional heating as shown in Table 1.

The ${ }^{1} \mathrm{H}$ NMR spectrum of $\mathbf{7} \mathbf{k}$ as a prototype for the benzaldehyde derivatives showed a multiplet peak in the range at $\delta 3.71-3.74 \mathrm{ppm}$ related to 4 methylene groups (4 $\mathrm{OCH}_{2}-$ ), another multiplet peak in the range at $\delta 3.75-$ $3.84 \mathrm{ppm}$ related to 4 methylene groups $\left(4 \mathrm{NCH}_{2}-\right)$, doublet at $\delta 7.36 \mathrm{ppm}$ for the two aromatic protons $\mathrm{H}-3$ and $\mathrm{H}-5$, doublet at $\delta 7.72$ for the twoaromatic protons $\mathrm{H}-2$ and $\mathrm{H}-6$, singlet at $\delta 7.82$ related to the $\mathrm{CH}(\mathrm{CH}=\mathrm{N}$ - group), and a broad singlet at $\delta 8.31$ for the $\mathrm{NH}$. The ${ }^{13} \mathrm{C}$ NMR spectrum of $7 \mathbf{k}$ showed absorption peak for the morpholine residue at $\delta 43.6$, $43.7,66.8$, and 66.9 related to $2 \mathrm{CH}_{2}-\mathrm{N}-\mathrm{CH}_{2}$, and $2 \mathrm{CH}_{2}-\mathrm{O}-$ $\mathrm{CH}_{2}$ respectively, absorption peaks at $\delta 128.2,128.9,132.8$, and $135.4 \mathrm{ppm}$ related to the aromatic carbons, and absorption peaks at $\delta 142.5$ and $165.4 \mathrm{ppm}$ for $\mathrm{C}=\mathrm{N}$.

The LC-MS of compound $7 \mathbf{k}$ using buffer A, $0.1 \%$ formic acid in $\mathrm{H}_{2} \mathrm{O}$, and buffer $\mathrm{B}, 0.1 \%$ formic acid in $\mathrm{CH}_{3} \mathrm{CN}$, in 


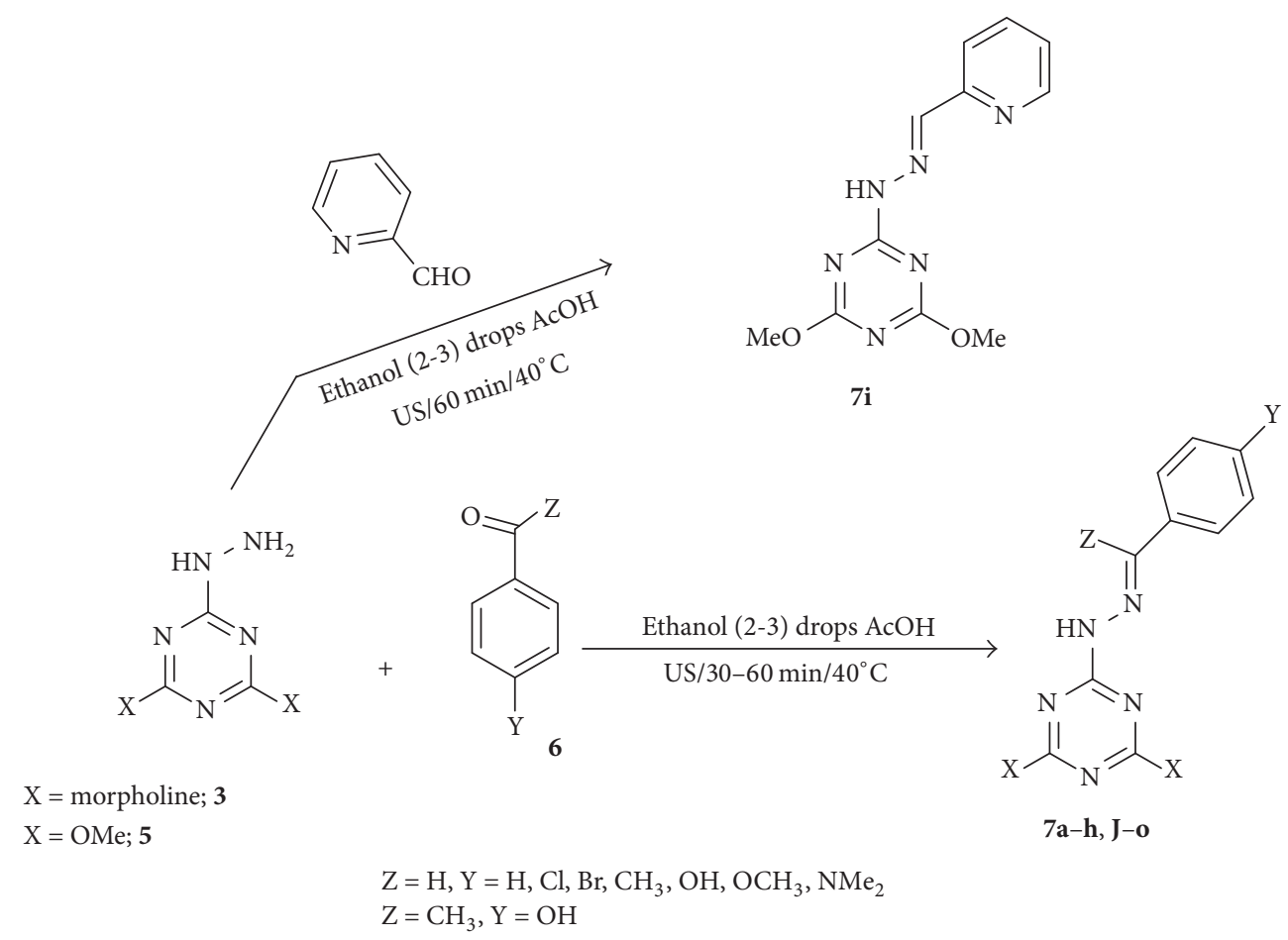

Scheme 3: Synthesis of 4,6-disubstituted-s-triazine-Schiff base derivatives and US: ultrasonic irradiation.<smiles>Clc1ccc(/C=N\Nc2nc(N/N=C/c3ccc(Cl)nc3N3CCOCC3)nc(N3CCOCC3)n2)cc1</smiles>

SCHEME 4: E- and Z-isomer of compound $\mathbf{7 k}$.

30 min showed one peak at $R_{t} 15.8 \mathrm{~min}$ with the expected mass $[\mathrm{M}+\mathrm{H}] 404(\mathrm{~m} / \mathrm{z}$ cacld. 403.87, Figure S13 in the Supplementary Material).

Compound $7 \mathbf{k}$ as an example could adopt two different geometrical isomers ( $E, Z$; Scheme 4). Therefore, $7 \mathbf{k}$ was demonstrated using molecular mechanics MM2 calculations. In addition, quantum chemical calculations were carried out with the GAUSSIAN 98 suite of programs. Geometry optimization was carried out using the DFT level (B3LYP/6-31G ${ }^{* *}$ ) of theory to assess the relative stability of the $E-Z$ isomeric species. Computed relative energies of $7 \mathbf{k}$ indicated that the $E$-isomer (total energy content $47.7837 \mathrm{kcal} / \mathrm{mol}$ ) is more stable than the $Z$ ones (total energy content $41.7295 \mathrm{kcal} / \mathrm{mol}$ ) by $6.0542 \mathrm{kcal} / \mathrm{mol}$. This observation agreed with our reported data in which the s-triazine hydrazone preferred the $E$-isomer rather than the $Z$ - ones [41].

As a prototype for acetophenone derivatives, the ${ }^{1} \mathrm{H}$ NMR spectrum of $7 \mathbf{o}$ showed a singlet peak at $\delta 2.24 \mathrm{ppm}$ for the methyl group of the acetophenone moiety, two multiplet peaks in the range $\delta 3.70-3.83 \mathrm{ppm}$ related to the 8 methylene groups of the two morpholino rings $\left(4-\mathrm{N}-\mathrm{CH}_{2}-\mathrm{CH}_{2}-\mathrm{O}\right)$, and a broad singlet at $\delta 8.01 \mathrm{ppm}$ related to the $\mathrm{NH}$, beside two doublets forming an $\mathrm{AB}$ system at $\delta 6.80(J=8.8 \mathrm{~Hz})$ and $7.698 \mathrm{ppm}(J=8.46)$ for the aromatic protons $\mathrm{H}-3, \mathrm{H}-5$, and $\mathrm{H}-2, \mathrm{H}-6$ respectively. The ${ }^{13} \mathrm{C}$ NMR spectrum showed absorption peak at $\delta 12.7$ related to methyl group and two peaks at $\delta 43.7$ and $66.9 \mathrm{ppm}$ related to the methylene groups $\left(-\mathrm{NCH}_{2}\right.$ and $\mathrm{O}-\mathrm{CH}_{2}$, resp.); the absorption peaks at $\delta 156.8$, $164.7,165.2$, and $165.4 \mathrm{ppm}$ were assigned for carbons of 
TABLE 2: Antimicrobial activity (zones of inhibition, $\mathrm{mm}$ ) and a minimum inhibitory concentration (MIC, $\mathrm{mg} / \mathrm{ml}$ ) of $7 \mathbf{i}$ and $7 \mathbf{k}$ comparing with several standard antimicrobial drugs.

\begin{tabular}{|c|c|c|c|c|c|c|c|c|c|c|c|}
\hline \multirow{2}{*}{\multicolumn{2}{|c|}{ Microorganisms }} & \multirow{2}{*}{\multicolumn{3}{|c|}{ Antifungal agent }} & \multirow{2}{*}{\multicolumn{3}{|c|}{ Antibacterial agents }} & \multicolumn{4}{|c|}{ Compounds } \\
\hline & & & & & & & & \multicolumn{2}{|c|}{$\mathrm{mM}$} & \multicolumn{2}{|c|}{$\mathrm{mg} / \mathrm{mL}$} \\
\hline & & Cy. ${ }^{*}$ & Co. & Ca. & To. & Ch. & $\mathrm{Fu}$. & $7 \mathbf{i}$ & $7 \mathbf{k}$ & $7 \mathbf{i}$ & $7 \mathbf{k}$ \\
\hline \multirow{7}{*}{ Bacteria } & $\mathrm{Sa}^{* *}$ & N.T & N.T & N.T & 14 & 30 & 23 & 15 & - & 0.10 & - \\
\hline & Sm. & N.T & N.T & N.T & - & 35 & 20 & 10 & - & 0.20 & - \\
\hline & Ec. & N.T & N.T & N.T & 20 & 40 & - & - & $<8$ & - & - \\
\hline & $\mathrm{Pa}$ & N.T & N.T & N.T & 15 & 13 & - & - & - & - & - \\
\hline & St. & N.T & N.T & N.T & 14 & 30 & - & - & $<8$ & - & - \\
\hline & $\mathrm{Nm}$ & N.T & N.T & N.T & 21 & 34 & 20 & 15 & 17 & 0.10 & 0.10 \\
\hline & $\mathrm{BL}$ & N.T & N.T & N.T & - & 35 & 20 & 20 & - & 0.05 & - \\
\hline \multirow{6}{*}{ Fungi } & Cp. & - & - & - & N.T $T^{* * *}$ & N.T & N.T & - & - & - & - \\
\hline & $\mathrm{Cn}$. & 32 & 25 & - & N.T & N.T & N.T & - & - & - & - \\
\hline & $\mathrm{Ca}$. & - & 46 & 20 & N.T & N.T & N.T & - & - & - & - \\
\hline & Pc. & 23 & 30 & - & N.T & N.T & N.T & - & - & - & - \\
\hline & An. & 22 & 23 & 20 & N.T & N.T & N.T & - & - & - & - \\
\hline & Fs. & 30 & 15 & - & N.T & N.T & N.T & - & - & - & - \\
\hline
\end{tabular}

* Cy: cycloheximide, Co: canesten, Ca: caspofungin, To: tobramycin, Ch: chloramphenicol, and Fu: fusidic acid. Sa ${ }^{* *}$ : Staphylococcus aureus ATCC 29213, Nm: Neisseria meningitides ATCC 1302, Sm: Streptococcus mutans ATCC 35668, EC: Escherichia coli ATCC 25922, Pa: Pseudomonas aeruginosa ATCC 27584, St: Salmonella typhimurium ATCC 14028, Cp: Candida parapsilosis ATCC 22019, Cn: Cryptococcus neoformans Wild strain, C: Candid albicans ATCC 60193, BL: Brevibacillus laterosporus Wild strain, Pc: Penicillium chrysogenum AUMC 9476, An: Aspergillus niger AUMC 8777, and Fs: Fusarium sp. N.T*** : not tested.

$\mathrm{C}=\mathrm{N}$ and the remaining five peaks at $\delta 115.3,127.8,128.2$, 130.9 , and $146.9 \mathrm{ppm}$ related to the aromatic carbons of the acetophenone moiety.

The LC-MS of compound 7o using buffer A, $0.1 \%$ formic acid in $\mathrm{H}_{2} \mathrm{O}$, and buffer $\mathrm{B}, 0.1 \%$ formic acid in $\mathrm{CH}_{3} \mathrm{CN}$, in 30 min showed one peak at $R_{t} 13.4$ min with the expected mass $[\mathrm{M}+\mathrm{H}] 400(\mathrm{~m} / \mathrm{z}$ calcd 399.46, Figure $\mathrm{S} 18$ in the Supplementary Material)

3.2. Biology. The antimicrobial activities of the prepared compounds against several pathogenic tested organisms are represented in Table 2 . The results revealed that only two compounds $7 \mathbf{i}$ and $7 \mathbf{k}$ from all the tested compounds were biologically active with different spectrum activity; on the other hand, they did not show any antifungal activity (Table 2). The results in Table 2 showed that $7 \mathbf{i}$ had good biological activity against $S$. aureus, $N$. meningitides, and tobramycin resistant $B$. laterosporus, while it had poor biological activity against $S$. mutans $(10 \mathrm{~mm})$. Compound $7 \mathbf{k}$ showed a good biological activity against $N$. meningitides $(17 \mathrm{~mm})$ and a very weak activity $(<8)$ against $E$. coli and $S$. typhimurium. The measured minimum inhibitory concentrations (MIC) of $7 \mathbf{i}$ that inhibited $S$. aureus, $S$ mutans, $N$. meningitides, and B. laterosporus were $0.10,0.20,0.10$ and $0.05 \mathrm{mg} / \mathrm{mL}$, respectively, while the MIC of $7 \mathbf{k}$ that inhibited $N$. meningitides was $0.10 \mathrm{mg} / \mathrm{mL}$.

It is obvious from Table 2 that a small structural variation in s-triazine ring may induce an effect on antibacterial activity. By observing the antibacterial activities of all the derivatives, it is interesting to note that the dimorpholino-4chlorophenyl-s-triazine derivatives $\mathbf{7 k}$ show good antibacterial activity against $N$. meningitides and weak activity against
E. coli and S. typhimurium. The other derivatives in the same family did not show any biological activity; this observation agreed with the reported data for substituted s-triazine [45]. The presence of pyridine ring (compound 7o) in place of the benzaldehyde ring (compound 7a) changed the biological activity significantly as observed from Table 2; on the other hand, all the tested compounds did not show any antifungal activity; this observation also agreed with the reported data for s-triazine derivatives [45].

\section{Conclusion}

The target products $(7 \mathbf{a}-\mathbf{o})$ were prepared using conventional heating and ultrasonic irradiation. The ultrasonic irradiation affords the products in higher yields and purity in shorter reaction time. The biological activity for the prepared compounds showed that only two compounds $7 \mathbf{i}$ (pyridine derivative) and $7 \mathbf{k}$ (4-chlorobenzaldehyde derivative) have biological activities against some Gram-positive and Gramnegative bacteria, while all tested compounds did not show any antifungal activity. It is obvious that changing the substituent in the s-triazine ring as well as the benzylidene moiety may affect the antimicrobial activity. Further investigations are run in our lab to get insights into the mode of action and the relation between the biological activity and substituent effect in the pattern in s-triazine.

\section{Competing Interests}

The authors declare no conflict of interests. 


\section{Acknowledgments}

The authors thank the Deanship of Scientific Research at King Saud University for funding this work through Prolific Research Group Program (PRG-1437-33; Saudi Arabia).

\section{References}

[1] C. Petrier, J. L. Luche, and J. L. Luche, Synthetic Organic Sonochemistry, Plenum Press, New York, NY, USA, 1998.

[2] J.-T. Li, W.-Z. Yang, S.-X. Wang, S.-H. Li, and T.-S. Li, "Improved synthesis of chalcones under ultrasound irradiation," Ultrasonics Sonochemistry, vol. 9, no. 5, pp. 237-239, 2002.

[3] K. Shibata, I. Katsuyama, M. Matsui, and H. Muramatsu, "Synthesis of ferrocenyl-substituted 3-cyano-2-methylpyridines," Bulletin of the Chemical Society of Japan, vol. 63, no. 12, pp. 37103712, 1990.

[4] P. Cintas and J.-L. Luche, "Green chemistry: the sonochemical approach," Green Chemistry, vol. 1, no. 3, pp. 115-125, 1999.

[5] T. J. Mason, "Ultrasound in synthetic organic chemistry," Chemical Society Reviews, vol. 26, no. 6, pp. 443-451, 1997.

[6] R. Cella and H. A. Stefani, "Ultrasound in heterocycles chemistry," Tetrahedron, vol. 65, no. 13, pp. 2619-2641, 2009.

[7] M. F. Mady, A. A. El-Kateb, I. F. Zeid, and K. B. Jorgensen, "Comparative studies on conventional and ultrasound-assisted synthesis of novel homoallylic alcohol derivatives linked to sulfonyl dibenzene moiety in aqueous media," Journal of Chemistry, vol. 2013, Article ID 364036, 9 pages, 2013.

[8] J.-T. Li, G.-F. Chen, W.-Z. Yang, and T.-S. Li, "Ultrasound promoted synthesis of 2-aroyl-1,3,5-triaryl-4-carbethoxy-4cyanocyclohexanols," Ultrasonics Sonochemistry, vol. 10, no. 3, pp. 123-126, 2003.

[9] J.-T. Li, G.-F. Chen, W.-Z. Xu, and T.-S. Li, "The Michael reaction catalyzed by KF/basic alumina under ultrasound irradiation," Ultrasonics Sonochemistry, vol. 10, no. 2, pp. 115-118, 2003.

[10] L. Ding, W. Wang, and A. Zhang, "Synthesis of 1,5-dinitroaryl1,4-pentadien-3-ones under ultrasound irradiation," Ultrasonics Sonochemistry, vol. 14, no. 5, pp. 563-567, 2007.

[11] W. T. Richards and A. L. Loomis, "The chemical effects of high frequency sound waves I. A preliminary survey," Journal of the American Chemical Society, vol. 49, no. 12, pp. 3086-3100, 1927.

[12] C. Leonelli and T. J. Mason, "Microwave and ultrasonic processing: now a realistic option for industry," Chemical Engineering and Processing: Process Intensification, vol. 49, no. 9, pp. 885900, 2010.

[13] G. Cravotto and P. Cintas, "Power ultrasound in organic synthesis: moving cavitational chemistry from academia to innovative and large-scale applications," Chemical Society Reviews, vol. 35, no. 2, pp. 180-189, 2006.

[14] T. J. Mason, A. J. Cobley, J. E. Graves, and D. Morgan, "New evidence for the inverse dependence of mechanical and chemical effects on the frequency of ultrasound," Ultrasonics Sonochemistry, vol. 18, no. 1, pp. 226-230, 2011.

[15] R. S. Yamgar, Y. R. Nivid, S. Nalawade, M. Mandewale, R. G. Atram, and S. S. Sawant, "Novel zinc(II) complexes of heterocyclic ligands as antimicrobial agents: synthesis, characterisation, and antimicrobial studies," Bioinorganic Chemistry and Applications, vol. 2014, Article ID 276598, 10 pages, 2014.

[16] M. C. Mandewale, B. R. Thorat, D. Shelke, R. Patil, and R. Yamgar, "Synthesis, characterization and fluorescence study of $N$-[(E)-(2-hydroxyquinolin-3-yl)methylidene]-1-benzofuran- 2-carbohydrazide and its metal complexes," Heterocyclic Letters, vol. 5, no. 2, pp. 251-259, 2015.

[17] M. C. Mandewale, B. R. Thorat, and R. S. Yamgar, "Synthesis and anti-mycobacterium study of some fluorine containing Schiff bases of quinoline and their metal complexes," Der Pharma Chemica, vol. 7, no. 5, pp. 207-215, 2015.

[18] D. N. Dhar and C. L. Taploo, "Schiff bases and their applications," Journal of Scientific \& Industrial Research, vol. 41, no. 8, pp. 501-506, 1982.

[19] P. Przybylski, A. Huczynski, K. Pyta, B. Brzezinski, and F. Bartl, "Biological properties of schiff bases and azo derivatives of phenols," Current Organic Chemistry, vol. 13, no. 2, pp. 124-148, 2009.

[20] M. Singh and N. Raghav, "Biological activities of hydrazones: a review," International Journal of Pharmacy and Pharmaceutical Sciences, vol. 3, no. 4, pp. 26-32, 2011.

[21] B. Kaya, Y. Özkay, H. E. Temel, and Z. A. Kaplancıklı, "Synthesis and biological evaluation of novel piperazine containing hydrazone derivatives," Journal of Chemistry, vol. 2016, Article ID 5878410, 7 pages, 2016.

[22] M. C. Mandewale, B. Thorat, D. Shelke, and R. Yamgar, "Synthesis and biological evaluation of new hydrazone derivatives of quinoline and their $\mathrm{Cu}(\mathrm{II})$ and $\mathrm{Zn}$ (II) complexes against Mycobacterium tuberculosis," Bioinorganic Chemistry and Applications, vol. 2015, Article ID 153015, 14 pages, 2015.

[23] G. Blotny, "Recent applications of 2,4,6-trichloro-1,3,5-triazine and its derivatives in organic synthesis," Tetrahedron, vol. 62, no. 41, pp. 9507-9522, 2006.

[24] B. Kolesinska and Z. J. Kaminski, "The umpolung of substituent effect in nucleophilic aromatic substitution. A new approach to the synthesis of $\mathrm{N}, \mathrm{N}$-disubstituted melamines (triazine triskelions) under mild reaction conditions," Tetrahedron, vol. 65, no. 18, pp. 3573-3576, 2009.

[25] G. R. Gustafson, C. M. Baldino, M.-M. E. O’Donnell et al., "Incorporation of carbohydrates and peptides into large triazine-based screening libraries using automated parallel synthesis," Tetrahedron, vol. 54, no. 16, pp. 4051-4065, 1998.

[26] S. S. Machakanur, B. R. Patil, D. S. Badiger, R. P. Bakale, K. B. Gudasi, and S. W. A. Bligh, "Synthesis, characterization and anticancer evaluation of novel tri-arm star shaped 1,3,5-triazine hydrazones," Journal of Molecular Structure, vol. 1011, pp. 121127, 2012.

[27] K. Srinivas, U. Srinivas, V. J. Rao, K. Bhanuprakash, K. H. Kishore, and U. S. N. Murty, "Synthesis and antibacterial activity of 2,4,6-tri substituted s-triazines," Bioorganic and Medicinal Chemistry Letters, vol. 15, no. 4, pp. 1121-1123, 2005.

[28] G. Nieddu and G. Giacomelli, "A microwave assisted synthesis of benzoxazoles from carboxylic acids," Tetrahedron, vol. 69, no. 2, pp. 791-795, 2013.

[29] C. Courme, N. Gresh, M. Vidal et al., "Synthesis of aryl phosphates based on pyrimidine and triazine scaffolds," European Journal of Medicinal Chemistry, vol. 45, no. 1, pp. 244-255, 2010.

[30] J. P. Raval, A. R. Rai, N. H. Patel, H. V. Patel, and P. S. Patel, "Synthesis and in vitro antimicrobial activity of N'(4-(arylamino)-6- (pyridin-2-ylamino)-1,3,5-triazin-2-yl)benzohydrazide," International Journal of ChemTech Research, vol. 1, no. 3, pp. 616-620, 2009.

[31] S. Nishigaki, F. Yoneda, H. Matsumoto, and K. Morinaga, "Synthetic antibacterials. I. Nitrofurylvinyl-s-triazine derivatives," Journal of Medicinal Chemistry, vol. 12, no. 1, pp. 39-42, 1968. 
[32] L. D. S. Yadav, K. N. Shukla, R. Dwivedi, and H. Singh, "Synthesis of new 1, 3, 4-oxadiazolo [3, 2-a]-s-triazine-5, 7dithiones and the dithionone analogues as potential antifungal agents," Indian Journal of Pharmaceutical Sciences, vol. 54, no. 1, pp. 33-37, 1992.

[33] D. H. Mahajan, C. Pannecouque, E. De Clercq, and K. H. Chikhalia, "Synthesis and studies of new 2-(coumarin-4yloxy)-4,6-(substituted)-S-triazine derivatives as potential antiHIV agents," Archiv der Pharmazie, vol. 342, no. 5, pp. 281-290, 2009.

[34] Z. Nie, C. Perretta, P. Erickson et al., "Structure-based design, synthesis, and study of pyrazolo[1,5-a] $[1,3,5]$ triazine derivatives as potent inhibitors of protein kinase CK2," Bioorganic and Medicinal Chemistry Letters, vol. 17, no. 15, pp. 4191-4195, 2007.

[35] Z. Nie, C. Perretta, P. Erickson et al., "Structure-based design and synthesis of novel macrocyclic pyrazolo[1,5-a] [1,3,5]triazine compounds as potent inhibitors of protein kinase CK2 and their anticancer activities," Bioorganic and Medicinal Chemistry Letters, vol. 18, no. 2, pp. 619-623, 2008.

[36] V. K. Pandey, S. Tusi, Z. Tusi, M. Joshi, and S. Bajpai, "Synthesis and biological activity of substituted 2,4,6-s-triazines," Acta Pharmaceutica, vol. 54, no. 1, pp. 1-12, 2004.

[37] A. Agarwal, K. Srivastava, S. K. Puri, and P. M. S. Chauhan, "Synthesis of substituted indole derivatives as a new class of antimalarial agents," Bioorganic and Medicinal Chemistry Letters, vol. 15, no. 12, pp. 3133-3136, 2005.

[38] V. R. Avupati, R. P. Yejella, V. R. Parala et al., "Synthesis, characterization and in vitro biological evaluation of some novel 1,3,5triazine-Schiff base conjugates as potential antimycobacterial agents," Bioorganic and Medicinal Chemistry Letters, vol. 23, no. 21, pp. 5968-5970, 2013.

[39] W. Zhu, Y. Liu, Y. Zhao et al., "Synthesis and biological evaluation of novel 6-hydrazinyl-2,4-bismorpholino pyrimidine and 1,3,5-triazine derivatives as potential antitumor agents," Archiv der Pharmazie, vol. 345, no. 10, pp. 812-821, 2012.

[40] A. El-Faham, S. M. Soliman, S. M. Osman et al., "One pot synthesis, molecular structure and spectroscopic studies (X-ray, IR, NMR, UV-Vis) of novel 2-(4,6-dimethoxy-1,3,5-triazin-2yl) amino acid ester derivatives," Spectrochimica Acta-Part A: Molecular and Biomolecular Spectroscopy, vol. 159, pp. 184-198, 2016.

[41] A. El-Faham, S. M. Soliman, H. A. Ghabbour et al., "Ultrasonic promoted synthesis of novel s-triazine-Schiff base derivatives; molecular structure, spectroscopic studies and their preliminary anti-proliferative activities," Journal of Molecular Structure, vol. 1125, pp. 121-135, 2016.

[42] S. T. Asundaria, S. A. Patel, K. M. Mehta, and K. C. Patel, "Synthesis, characterization and antimicrobial studies of some novel 1,3,4-thiadiazolium-2-thiolate derivatives," South African Journal of Chemistry, vol. 63, pp. 141-144, 2015.

[43] D. G. Gerbig Jr., J. E. Ndong, and H. Aubihl, "A new twist to the kirby-bauer antibiotic susceptibility test activity-increasing antibiotic sensitivity of pseudomonas fluorescens through thermal stress," Journal of Microbiology and Biology Education, vol. 14, no. 2, pp. 269-270, 2013.

[44] S. J. Cavalieri, Manual of Antimicrobial Susceptibility Testing, American Society for Microbiology, 2005.

[45] K. Srinivas, U. Srinivas, K. Bhanuprakash, K. Harakishore, U. S. N. Murthy, and V. Jayathirtha Rao, "Synthesis and antibacterial activity of various substituted s-triazines," European Journal of Medicinal Chemistry, vol. 41, no. 11, pp. 1240-1246, 2006. 

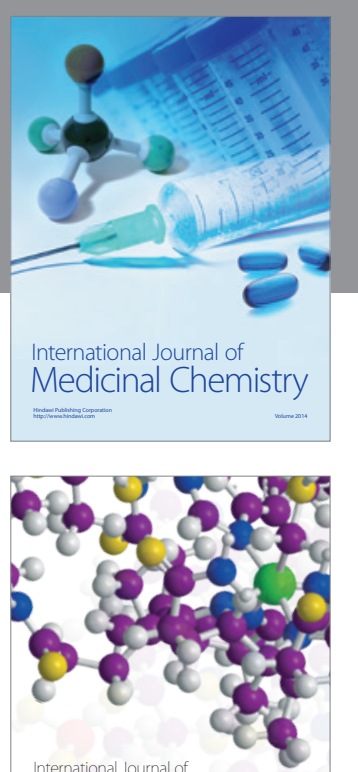

Carbohydrate Chemistry

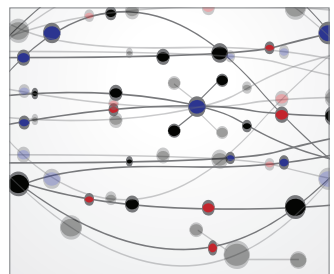

The Scientific World Journal
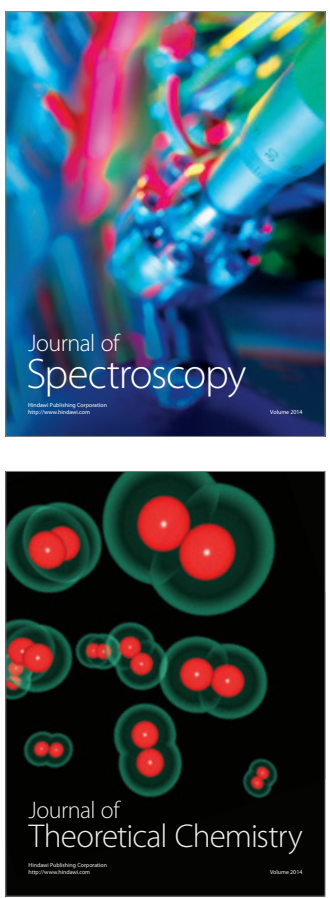
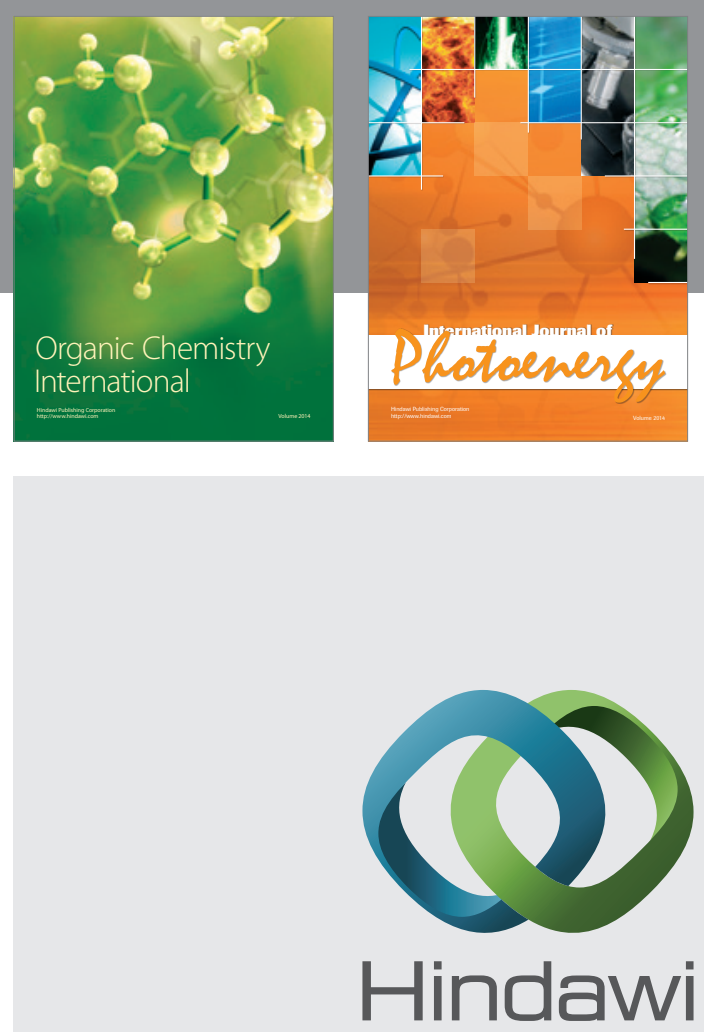

Submit your manuscripts at

http://www.hindawi.com

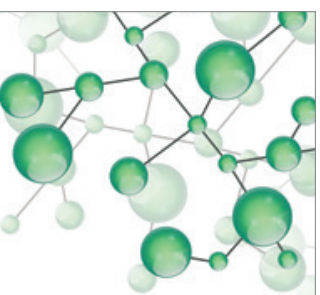

International Journal of

Inorganic Chemistry

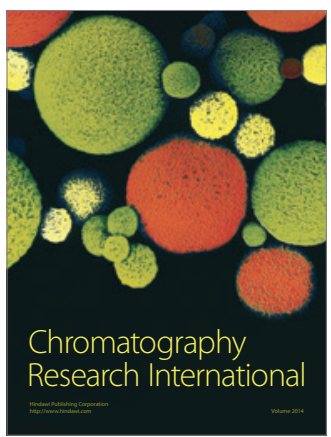

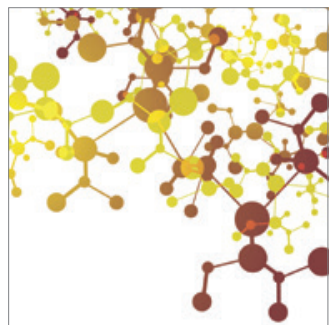

Applied Chemistry
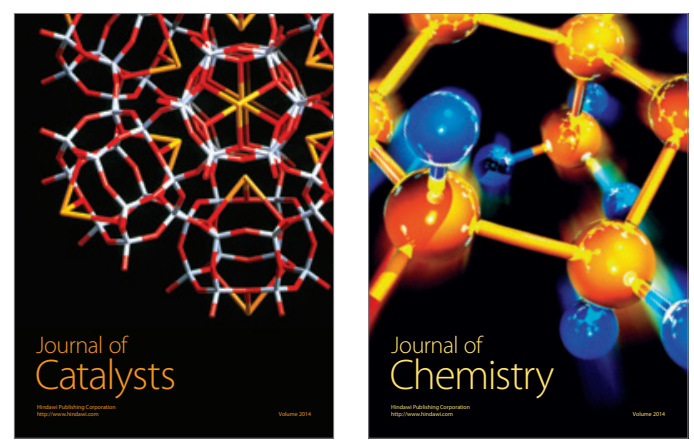
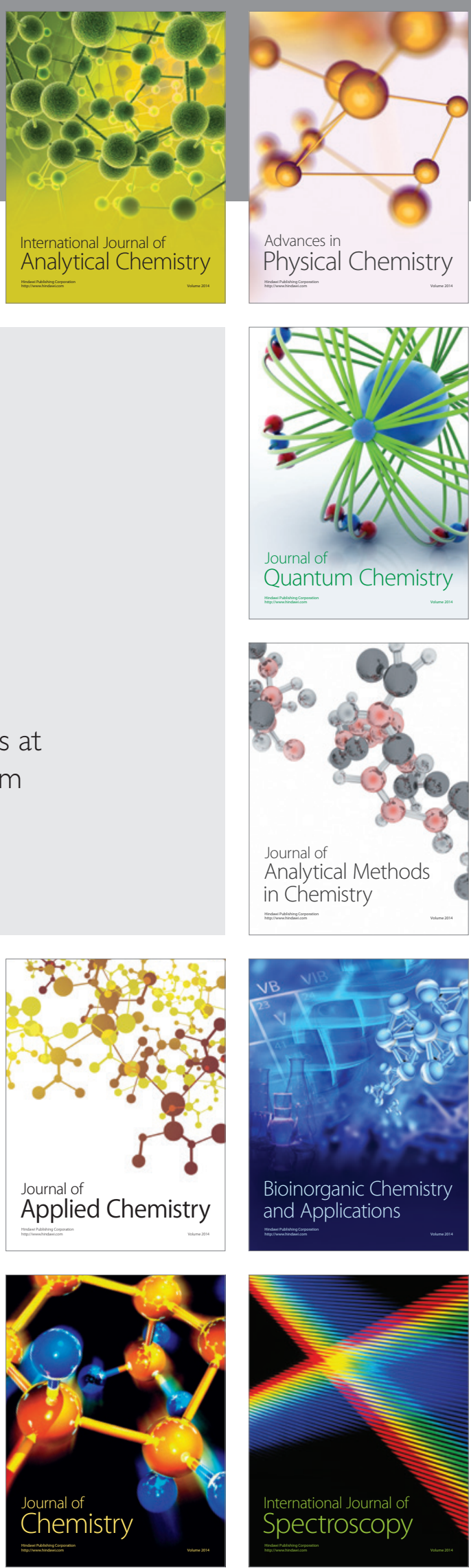